\title{
Postoperative Complications of Thyroidectomy
}

\author{
Bode Rovena ${ }^{1}$, Celiku Etmond ${ }^{2}$ \\ ${ }^{1}$ Department of General Surgery, "Mother Teresa" University Hospital Center, Rruga e Dibres, 1025, Tirana, Albania
}

\begin{abstract}
Thyroidectomy as a frequent and relatively safety surgical procedure is associated with a minimal rate of complications. Modern thyroidectomy, centers on potential morbidity from the procedure with emphasis on prevention of injury to closely investing structures such as the parathyroid glands and the recurrent laryngeal nerves. This study was a prospective review of all thyroidectomies performed at the Department of General Surgery in the University Hospital Center "Mother Teresa" of Tirana during January 2010January 2013. It is focused in the complications of postoperative period of thyroidectomy, especially hypocalcemia and laryngeal nerve palsy. For each case, presence or absence of individual surgical complications including wound infection, postoperative hemorrhage, fistula formation, hypocalcemia, and recurrent laryngeal nerve injury was determined. Besides recommending particular care with intraoperative identification and preservation of the parathyroids and recurrent laryngeal nerve, it may also be prudent to examine the thyroidectomy specimens of these patients carefully with the intention of identifying healthy parathyroid glands that may be suitable for autotransplantation.
\end{abstract}

Keywords: hypocalcemia, hypoparathyroidism, laryngeal nerve palsy, parathyroid

\section{Introduction}

Thyroidectomy as a frequent and relatively safety surgical procedure is associated with a minimal rate of complications. In fact the focus of modern thyroidectomy, centers on potential morbidity from the procedure with emphasis on prevention of injury to closely investing structures such as the parathyroid glands and the recurrent laryngeal nerve(1-5). As opposed to unilateral thyroid lobectomy, total thyroidectomy carries potential risk to all 4 parathyroid glands and both recurrent laryngeal nerves. Whereas parathyroid injury during unilateral thyroid lobectomy is unlikely to result in hypocalcemia, postoperative hypocalcemia is a significant risk after total thyroidectomy. Similarly, although unilateral recurrent laryngeal nerve injury during thyroid lobectomy may have a significant impact on voice quality, bilateral recurrent laryngeal nerve injury during a total thyroidectomy has more serious airway consequences $(4,5)$. Therefore, it is essential to quantify the incidences of postoperative hypoparathyroidism and recurrent laryngeal nerve injury, and to identify potential predictive clinical factors for these morbidities. Such information is important to properly counsel patients and determine preoperative risks for patients considering total thyroidectomy. To minimize morbidity it is recommended that these anatomic structures be recognize during surgery, moreover the surgeon should keep in mind anatomic variations of parathyroid glands and recurrent laryngeal nerves $(6,7)$. Experience of the operating surgeon and extent of surgery are important for the complication rate in thyroid surgery(1).

Inadvertent parathyroidectomy is a potential complication which occurrence varies among surgeons depending on individual experience(8-11).During thyroidectomy, parathyroid preservation can be achived by careful dissection directly on the thyroid capsule and separating the parathyroid glands gently from the thyroid gland. Although the removal of one or more glands may not necessarily have any clinical relevance, for some authors(12-15), it has been a risk factor for postthyroid hypocalcemia.

\section{Methods}

This study was a prospective review of all thyroidectomies (unilateral and bilateral) performed at the Department of General Surgery in the University Hospital Center "Mother Teresa" of Tirana, during January 2010- January 2013. Patients demographics and data regarding preoperative diagnosis and surgical procedure were collected. Patients undergoing concomitant central neck lymph nodal dissection were included in the study whereas those who had undergone repeated surgical procedures for recurrent or persistent thyroid desease and patients who had undergone intentional parathyroidectomy, were excluded from the study. The following parameters were recorded for all patients: age, sex, preoperative diagnosis, postoperative diagnosis, thyroid hormonal status, number of parathyroid glands identified and spared during surgery, autotransplantation of parathyroid glands and final histologic report. For each case, presence or absence of individual surgical complications including wound infection, postoperative hemorrhage, fistula formation, hypocalcemia, and recurrent laryngeal nerve injury was determined.

\subsection{Surgical Technique}

The neck was explored through a collar incision or a transverse cervicotomy under general anesthesia, with/without division of the strap muscles. The surgical technique employed in most cases in the present series was a capsular dissection according the "Delbridge technique", involves hugging the gland and dividing the tertiary branches of the vessels while dissecting the parathyroid glands free from the thyroid gland, with reflection of parathyroid glands and/or parathyroid-containing tissue off the thyroid gland, without disruption of parathyroid blood supply(13). After mobilization of the thyroid gland the recurrent laryngeal nerve and inferior thyroid artery were displayed on both sides and a meticulous search was made for the parathyroid glands. Criteria used for positively identification of parathyroid glands were characteristic colour (caramel yellow-brown) and consistency (firm, well-defined, and non-friable), with or 


\section{International Journal of Science and Research (IJSR) \\ ISSN (Online): 2319-7064}

Index Copernicus Value (2013): 6.14 | Impact Factor (2014): 5.611

without colour change with devascularisation in certain cases. Positive identification of parathyroid was made only where the senior author was confident that parathyroid was unequivocally identified. Cases of "possible" parathyroids were considered to not have been positively identified. Unequivocally devascularized parathyroid glands were removed routinely for immediate autotransplantation into a pocket fashioned in the ipsilateral sternocleidomastoid muscle.

Definitive pathologic study was obtained for all resected tissues. Pathology reports were evaluated for the presence and the number of parathyroid glands inadvertently removed with the thyroid specimen and the location of the parathyroid gland (extracapsular, intracapsular or intrathyroidal).

Postoperative complications mainly temporary or permanent hypocalcemia, vocal cord paralysis, hemorrhage and infection were noted. Data on postoperative clinical hypocalcemia were searched. The presence and the type of hypocalcemic signs were recorded by a surgeon, together with the evaluation of the Chvostek sign.Serum calcium levels were measured on the first day after surgery. Patients were classified as having transient hypocalcemia if they were normocalcemic 2 weeks after stopping all follow-up medications. Postoperative hypocalcemia requiring treatment and associated with a low parathyroid hormone level, more than 1 year after surgery was classified as permanent.

\section{Results}

A total of 137 patients(113 female and 24 male) who underwent thyroidectomies in our department and satisfied the inclusion and exclusion criteria were included in the study. Their mean age was 44.56 years (range 13-78). The indications for surgery are listed in Table 1 . The most common indications for surgery were benign goiter and hyperthyroidism, accounting respectively for 71(51.8\%) and $49(35.7 \%)$ of cases. The thyroid function was in reference range in $88(64.233 \%)$ patients. Hyperthyroidism was exhibited by 49 patients $(35.7 \%)$. All 49 patients with hyperthyroidism were treated with methimazole and $\beta$ blockers and had normal thyroid function before the surgery. The primary thyroid lesion was benign in 108 patients (78.8\%) and malignant in the other 29 (21.2\%), documented preoperatively by fine-needle aspiration cytologic findings. Surgical procedures included total lobectomy and isthmusectomy in $27(19.7 \%$ ) patients, subtotal thyroidectomy in $57(41.6 \%)$ patients and total thyroidectomy in 53(38.7\%)patients(Table 1). Thirteen (13) patients underwent an associated nodal dissection along with total thyroidectomy, and 11 patients $(8 \%)$ received parathyroid reimplantation.
Table 1

\begin{tabular}{lr} 
Characteristic & Value(\%) \\
\hline Demographics & \\
Female sex & $113(82.5)$ \\
Age mean range & $44.56(13-78)$ \\
Indication for surgery & \\
Multinodular benign goiter & $71(51.8 \%)$ \\
Hyperthyroidism & $49(35.7 \%)$ \\
Solitary thyroid nodule & $9(6.6 \%)$ \\
Thyroid cancer & $8(5.8 \%)$ \\
Type of surgical procedure & \\
Total thyroidectomy & $53(38.7 \%)$ \\
Subtotal thyroidectomy & $57(41.6 \%)$ \\
Lobectomy and isthmusectomy & $27(19.7 \%)$ \\
Neck lymphnode dissection & $13(9.5 \%)$ \\
Parathyroid autotransplantation & $11(8 \%)$ \\
Thyroid pathologic characteristic & \\
Benign tumor & $108(78.8 \%)$ \\
Malignant neoplasm & $29(21.2 \%)$
\end{tabular}

Five patients $(3.6 \%)$ encountered a postoperative hematoma or hemorrhage, 2 patients $(1.45 \%)$ experienced a postoperative wound infection, and one patient had a kilorrhea. Overall, 23 patients $(16.7 \%)$ were identified with postoperative hypocalcemia. Eight patients (5.8\%) were diagnosed as having an associated unilateral vocal cord paralysis and 2 patients $(1.45 \%)$ had bilateral vocal cord paralysis(Table 2).

Table 2: Postthyroidectomy complications

\begin{tabular}{|c|c|}
\hline Complications & Total $\quad(\%)$ \\
\hline Hypocalcemia & $23 \quad(16.7 \%)$ \\
\hline Unilateral vocal cord paralysis & $8 \quad(5.8 \%)$ \\
\hline Bilateral vocal cord paralysis & $(1.45 \%)$ \\
\hline Hemorrhage & $(3.6 \%)$ \\
\hline Wound infection & $\begin{array}{ll}2 & (1.45 \%) \\
\end{array}$ \\
\hline Kilorrhea & $(0.73 \%)$ \\
\hline
\end{tabular}

During the intervention, the frequencies of parathyroid glands identified were as follows: 4 glands in 70 patients, 3 in 39 patients, 2 in 23 patients, and 1 in 4 patients. During or following resection of the thyroid gland and after carefully examining the surgical specimen intraoperatively, an inadvertently removed parathyroid gland was recognized in 11 of 137 patients $(8 \%)$. Seven of these were in the extracapsular location $(63.63 \%)$ and 4 in the intracapsular location $(36.37 \%)$. All of them had 1 parathyroid gland $(100 \%)$, which was salvaged. Parathyroid autotransplantation in the sternocleidomastoid muscle was then performed in all 11 patients at the time of thyroid surgery. Postoperative hypocalcemic signs were noticed in $8(72.72 \%)$ of them.

\subsection{Histopathologic Result}

Histopathologic examination of the resected thyroid revealed the presence of benign thyroid disease and thyroid malignant neoplasms in 100 patients $(73 \%)$ and 37 patients $(27 \%)$, respectively. Overall, 17 of the 137 patients (12.4\%) were found to have had inadvertent removal of parathyroid tissue . All of them had 1 parathyroid gland in their specimens. The examination of the location of the parathyroid tissue within the thyroid specimen showed that of the inadvertently removed parathyroid glands, $9(53 \%)$ were found in the extracapsular location, defined as lying outside the thyroid 


\section{International Journal of Science and Research (IJSR) \\ ISSN (Online): 2319-7064}

Index Copernicus Value (2013): 6.14 | Impact Factor (2014): 5.611

capsule; $4(23.5 \%)$ were seen in the intracapsular location, defined as being encased within the thyroid capsule or lying just beneath the capsule; and $4(23.5 \%)$ were located in the intrathyroidal position, defined as being completely surrounded by thyroid parenchyma(Table 3 ). In 16 patients of this group, the excised parathyroid was normal, only in one patient it resulted a parathyroid carcinoma. From the 17 patients with inadvertent removal of parathyroid gland, only 15 of them showed hypocalcemic signs postoperatively. The careful inspection of the thyroid specimen intraoperatively allowed reimplantation of inadvertently removed parathyroid glands during thyroidectomies in our series of patients, allowing a statistically significant reduction of the percentage of parathyroid glands discovered by the pathologist on the final surgical specimen (from $12.4 \%$ [11/137] to $8 \%$ [17/137]; $P=0.49,1$-sided $t$ test).

Table 3: Characteristics of inadvertently removed parathyroid glands

\begin{tabular}{lr} 
Characteristics & Value \\
\hline Inadvertent parathyroidectomy & $17(8 \%)$ \\
1 parathyroid gland removed & $17(100 \%)$ \\
Location of removed parathyroid glands & \\
Intrathyroidal & $4(23.5 \%)$ \\
Intracapsular & $4(23.5 \%)$ \\
Extracapsular & $9(53 \%)$ \\
Normal histopathology of removed glands & $16(94 \%)$
\end{tabular}

Hypocalcemia was transient in all patients; in all of them, the symptoms resolved within the 6- to 8-week period following thyroid surgery, and the supplementation therapy was stopped. Transient vocal cord paresis (by laryngoscopy) was observed in 3 patients with complete recuperation 2 to 3 months after surgery.

\section{Comments}

Postoperative hypocalcemia after total thyroidectomy has been reported to range from $1 \%$ to $40 \%(15-20)$. The incidence rate of hypocalcemia during thyroid surgery was $16.78 \%$ in our patients, which compares favorably with rates reported in the literature. Not unexpectedly, total thyroidectomy was strongly associated with postoperative hypocalcemia (incidence, $15.8 \%$ ) when compared with other forms of thyroid surgery. The incidence rate of inadvertent parathyroidectomy during thyroid surgery was $12.4 \%$ in our patients, which compares favorably with rates reported in the literature: $8 \%-19 \%(20-22)$. Unintentional parathyroidectomy was associated with an increased risk of postoperative hypocalcemia, 15 of 17 patients. The careful inspection of the thyroid specimen intraoperatively allowed reimplantation of inadvertently removed parathyroid glands during thyroidectomies in our series of patients, this retrieval being statistically significant (from $12.4 \%$ [11/137] to $8 \%$ [17/137], $P=.049 ; 1$-sided $t$ test). Lee et al ( 6) described their experience reporting an incidence rate of $11 \%$ for inadvertent parathyroid removal during thyroidectomy. Sasson et al (7) reported an incidence rate of $15 \%$ in a series of 141 thyroidectomies. In all cases, only 1 parathyroid gland was inadvertently resected with the thyroid (there was an incidence rate of $100 \%$ for removal of 1 gland in our study). Furthermore, despite some controversy, most authors agree that identifying parathyroid glands during thyroid surgery can result in a lower incidence of inadvertent parathyroidectomy and resulting hypocalcemia. However, dissection in search of all parathyroid glands during thyroid surgery is unwarranted and may be hazardous(9).

Familiarity with the anatomy of parathyroid glands and their blood supply is necessary to prevent inadvertent injury or devascularization or resection of the parathyroid parenchyma. Although there is great variability in their number, most commonly there are 4 glands commonly located extracapsularly on the posterior surface of the thyroid gland. They can, however, also be found within the thyroid capsule or, rarely, within the substance of the thyroid gland itself. The incidence of intrathyroidal location of parathyroid glands is approximately $0.2 \%$ according to autopsy studies, but this incidence rises to $2 \%$ to $5 \%$ in patients with primary hyperparathyroidism and up to $11 \%$ in those with persistent or recurrent hyperparathyroidism. In our study it was $0.88 \%$. In our study, the inadvertently resected parathyroid was intrathyroidal in $23.5 \%$ of cases. Obviously, in these cases, improvements in surgical technique cannot eliminate the risk of inadvertent parathyroidectomy; therefore, inadvertent parathyroidectomy may occur even in the hands of more experienced thyroid surgeons.

In our review of thyroidectomies with inadvertent excision of parathyroid tissue within the thyroid specimen, we noted that in most cases $(76.5 \%)$ the parathyroid tissue was found either in the extracapsular or intracapsular positions. It is conceivable that the parathyroid gland tissue in the extracapsular and intracapsular positions would be in locations where it could be identified and preserved during the surgery. Possibly, more careful examination of the thyroid capsule during and after thyroidectomy would decrease this incidence.

Following resection of the thyroid gland in our series of patients, and after examining the surgical specimen intraoperatively, an inadvertently excised parathyroid was recognized and autotransplanted after confirmation by frozen section analysis in 11 patients. In our series of patients, careful examination of the thyroid specimen was conducted intraoperatively, which resulted in identification of $32 \%$ $(11 / 28)$ of inadvertently removed parathyroid glands. The $23.5 \%$ of parathyroid tissue that was found in an intrathyroidal location would have been more difficult to preserve even with more careful dissection or inspection.

Published rates of recurrent laryngeal nerve injury vary widely in the literature. However, it is generally believed that recurrent laryngeal nerve injury, either temporary or permanent, is likely to occur in approximately $1 \%$ of unilateral lobectomy cases and $2 \%$ to $3 \%$ of total thyroidectomy cases(23-27)._In our study $10(7.15 \%)$ patients resulted with laryngeal nerve palsy, all of them in total/subtotal thyroidectomies. Total thyroidectomy carries an increased risk for recurrent laryngeal nerve palsy, not only because both recurrent laryngeal nerves are placed at risk, but also likely because patients undergoing total thyroidectomy often have more advanced disease. 


\section{International Journal of Science and Research (IJSR) \\ ISSN (Online): 2319-7064}

Index Copernicus Value (2013): 6.14 | Impact Factor (2014): 5.611

Besides recommending particular care with intraoperative identification and preservation of the parathyroids and recurrent laryngeal nerve, it may also be prudent to examine the thyroidectomy specimens of these patients carefully with the intention of identifying healthy parathyroid glands that may be suitable for autotransplantation.

\section{References}

[1] Lin DTPatel SGShaha ARSingh BShah JP Incidence of inadvertent parathyroid removal during thyroidectomy. Laryngoscope $2002 ; 112$ (4) 608-611 PubMed Link to Article

[2] Bergamaschi RBecouran GRonceray GArnaud JP Morbidity of thyroid surgery. Am J Surg 1998;176 (1) 71- 75PubMed Link to Article

[3] Reeve TThompson NW Complications of thyroid surgery: how to avoid them, how to manage them and observations on their possible effect on the whole patient. World $\quad J \quad$ Surg $\quad 2000 ; 24 \quad$ (8) 971-975 PubMed Link to Article

[4] Harness JKOrgan CHJThompson NW Operative experience of US general surgery residents in thyroid and parathyroid disease. Surgery. 1995;118:1063-1070.

[5] Bhattacharyya NFried MP Benchmarks for mortality, morbidity and length of stay for head and neck surgical procedures. Arch Otolaryngol Head Neck Surg.2001;127:127-132

[6] Lee NJBlakey JDBhuta SCalcaterra TC Unintentional parathyroidectomy during thyroidectomy. Laryngoscope 1999;109 (8) 1238- 1240 PubMed Link to Article

[7] Sasson ARPingpank JF JrWetherington RWHanlon ALRidge JA Incidental parathyroidectomy during thyroid surgery does not cause transient symptomatic hypocalcemia. Arch Otolaryngol Head Neck Surg 2001;127 (3) 304-308PubMed Link to Article

[8] Loré JM JrPruet CW Retrieval of the parathyroid glands during thyroidectomy. Head Neck Surg 1983;5 (3) 268-269PubMed Link to Article

[9] Sakorafas GHStafyla VBramis CKotsifopoulos NKolettis TKassaras G Incidental parathyroidectomy during thyroid surgery: an underappreciated complication of thyroidectomy. World J Surg 2005;29 (12) 1539- 1543 PubMed Link to Article

[10] Akerström GMalamaeus JBergstrom R Surgical anatomy of human parathyroid glands. Surgery 1984;95 (1) 14-21PubMed

[11]Abboud B Topographic anatomy and arterial vascularization of the parathyroid glands: practical application. Presse Med 1996;25 (25) 1156- 1161 PubMed

[12] Sakorafas GHStafyla VBramis CKotsifopoulos NKolettis TKassaras G Incidental parathyroidectomy during thyroid surgery: an underappreciated complication of thyroidectomy. World J Surg 2005;29 (12) 1539- 1543 PubMed Link to Article

[13] McHenry CRSperoff TWentworth DMurphy T Risk factors for postthyroidectomy hypocalcemia. Surgery 1994;116 (4) 641- 648 PubMed

[14]Pattou FCombemale FFabre S Hypocalcemia following thyroid surgery: incidence and prediction of outcome. World J Surg

PubMed Link to Article

[15] Delbridge L, Reeve TS, Khadra M, Poole AG. Total thyoidectomy: the technique of capsular dissection. Austr N Z J Surg. 1992 Feb;62(2):96-9.

[16] Mishra AAgarwal GAgarwal AMishra SK Safety and efficacy of total thyroidectomy in hands of endocrine surgery trainees. Am J Surg. 1999; 178:377-380.

[17] Pappalardo GGuadalaxara AFrattaroli FMIllomei GFalaschi P Total compared with subtotal thyroidectomy in benign nodular disease: personal series and review of published reports. Eur $J$ Surg. 1998;164:501-506.

[18]Bergamaschi RBecouarn GRonceray JArnaud JP Morbidity of thyroid surgery. Am J Surg. 1998;176:71-75.

[19] Szubin LKacker AKakani RKomisar ABlaugrund $S$ The management of post-thyroidectomy hypocalcemia. Ear Nose Throat J.1996;75:612-614.

[20]Flynn MBLyons KJTarter JWRagsdale TL Local complications after surgical resection for thyroid carcinoma. Am J Surg. 1994;168:404-407

[21] NJBlakey JDBhuta SCalcaterra TC Unintentional parathyroidectomy during thyroidectomy. Laryngoscope 1999;109 (8) 1238- 1240 PubMed Link to Article

[22] Sasson ARPingpank JF JrWetherington RWHanlon ALRidge JA Incidental parathyroidectomy during thyroid surgery does not cause transient symptomatic hypocalcemia. Arch Otolaryngol Head Neck Surg 2001;127 (3) 304- 308 PubMed Link to Article

[23]Loré JM JrPruet CW Retrieval of the parathyroid glands during thyroidectomy. Head Neck Surg 1983;5 (3) 268-269 PubMed Link to Article

[24]Bergamaschi RBecouarn GRonceray JArnaud JP Morbidity of thyroid surgery. Am J Surg. 1998;176:71-75.

[25] Szubin LKacker AKakani RKomisar ABlaugrund $\mathrm{S}$ The management of post-thyroidectomy hypocalcemia. Ear Nose Throat J.1996;75:612-614.

[26] Sasson ARPingpank Jr JFWetherington RWHanlon ALRidge JA Incidental parathyroidectomy during thyroid surgery does not cause transient symptomatic hypocalcemia. Arch Otolaryngol Head Neck Surg. 2001;127:304-308.

[27] Prim MPDe Diego JIHardisson DMadero RGavilan $\mathrm{J}$ Factors related to nerve injury and hypocalcemia in thyroid gland surgery. Otolaryngol Head Neck Surg.2001;124:111-114.

\section{Author Profile}

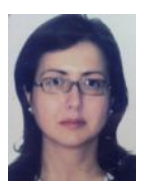

Rovena Bode (Corresponding author) Finished the study in Medecine University of Tirana, Albania in 2003. Graduaded as General Surgeon in 2009 in the General Surgery Department, "Mother Tereza", University hospital Center, Tirana, Albania. Medical Emergency Training in Torino, Italy in 2010. During 2011 fellowship in Laparoscopic Surgery and breast surgery (oncology surgery) in Naples, Italy. Actually General Surgeon in the Surgery Department "Mother Tereza "Universitary Hospital Center of Tirana, Albania. 